\title{
Actividad investigadora en medicina interna en Galicia.
}

\section{Quo vadis?}

\section{Research activity in internal medicine in Galicia}

\author{
Javier de la Fuente Aguado ${ }^{1}$, Arturo González Quintela ${ }^{2}$ \\ ${ }^{1}$ Hospital POVISA SA, Vigo. \\ ${ }^{2}$ Complexo Hospitalario Universitario de Santiago (CHUS). SERGAS. Santiago
}

\begin{abstract}
El programa formativo de la especialidad de Medicina Interna viene definido en la Orden SCO/227/2007, de 24 de enero. En ella se establecen las competencias que deben ser adquiridas por los futuros especialistas tanto en el ámbito asistencial como en el docente e investigador. Respecto a la investigación, textualmente dice que el residente en formación "debe iniciarse en el conocimiento de la metodología de la investigación" y "debe adquirir los conocimientos necesarios para realizar un estudio de investigación" $n^{\prime \prime}$. Este proceso se entiende como un continuum a lo largo de la residencia que se retroalimenta a sí mismo: a medida que se profundiza en la investigación se adquieren conocimientos y se desarrolla una mentalidad crítica que permite conocer las limitaciones o sesgos metodológicos, desgranar los resultados y dimensionar en su justa medida las conclusiones de los trabajos publicados por otros autores, o en palabras de la orden arriba citada "saber evaluar críticamente la literatura científica". Sin embargo, esta orden tiene un carácter orientador y, por lo tanto, no obliga o fija objetivos concretos, de ahí la enorme variabilidad observada en la actividad investigadora entre los distintos hospitales y comunidades autónomas de nuestro país.
\end{abstract}

El trabajo de Íñiguez et $a^{2}{ }^{2}$ publicado en este nuevo número de Galicia Clínica se suma y complementa al publicado con anterioridad por Rabuñal et $\mathrm{al}^{3}$, del mismo centro y servicio sanitario, reflejando el marcado y conocido interés de este grupo por las actividades formativas y de investigación dentro de la especialidad. El principal hallazgo es la constatación de una deficitaria actividad investigadora de los residentes de Medicina Interna de Galicia que se agudiza a medida que aumenta la dimensión del evento (congreso autonómico, nacional o internacional) o los requerimientos editoriales (publicaciones en revistas). De hecho, se observa una enorme brecha entre las comunicaciones a congresos y las publicaciones en revistas, que coincidiendo con los autores, puede ser debida a que no posean la calidad científica suficiente o a que no se efectúe el esfuerzo adicional requerido para publicar.
De manera sorprendente, no parece haber una correlación entre el año de residencia y la actividad investigadora, como ocurre en otras comunidades en las que esta actividad aumenta de forma marcada en los últimos años de la residencia ${ }^{4}$. Este hecho posiblemente sea debido a la menor tasa de respuesta de los residentes de tercer a quinto año y, en menor medida, a la escasa o nula participación en la encuesta de centros con una conocida trayectoria en investigación, como el Hospital Universitario de Santiago y el Hospital Xeral-Cíes de Vigo, respectivamente.

Como los autores acertadamente comentan en la discusión, el trabajo tiene una serie de limitaciones. En primer lugar, se trata de un trabajo efectuado a partir de una encuesta remitida a los tutores de residentes que, a su vez, se la entregaron a los residentes para que la completaran, sin que se pueda certificar que la información vertida en ella haya sido completa y verificada.

El segundo punto, motivo de reflexión es la tasa de participación: sólo contestaron dos tercios de los residentes, pero cuando se disgrega por año de residencia es llamativo que no alcanza la mitad de los residentes en formación de $3^{\circ}, 4^{\circ}$ y $5^{\circ}$ año. Esta escasa respuesta es en cierto modo un reflejo de la limitada implicación e importancia que los residentes otorgan a las actividades promovidas por la SOGAMI, como ya previamente pusieron de manifiesto Meijide et $\mathrm{al}^{5}$.

La producción científica de los residentes suele ser paralela a la del servicio docente donde se está formando y, por ello, el mayor peso de la responsabilidad recae en este último. Pero ¿cuáles son las principales barreras para que esta actividad investigadora sea tan deficiente? y, sobre todo, ¿qué se puede hacer para fomentarla?

Las encuestas suelen revelar siempre las mismas razones y tópicos para no investigar: poca formación específica en investigación, escaso impacto o importancia de lo investigado, situación desfavorable respecto a otros grupos con líneas de investigación 


\section{"la investigación requiere esfuerzo, dedicación, constancia, tenacidad y, sobre todo, dotes de observación y curiosidad suficientes}

para generar preguntas"

claramente definidas o establecidas, sobrecarga asistencial y escaso reconocimiento por este hecho.

$Y$ es que, efectivamente, la investigación requiere esfuerzo, dedicación, constancia, tenacidad y, sobre todo, dotes de observación y curiosidad suficientes para generarse preguntas (hipótesis u objetivos) y la manera de intentar responderlas (método científico). Precisa, pues, como la mayor parte de las actividades científicas creativas de actitud y de aptitud. Esta última requiere la integración de una sólida base de conocimientos científicos sobre el objeto de estudio y formación adecuada en metodología de la investigación. Sin embargo, una de las limitaciones a la que de forma reiterada recurren los residentes es el escasa formación que tienen sobre cómo planear o desarrollar un estudio de investigación, ya que durante la carrera universitaria no hay una materia que verse o trate de forma específica sobre este tema. En la formación pregrado se adquieren, aunque lamentablemente pocas veces se aprenden, conocimientos sobre epidemiología y estadística, herramientas imprescindibles, aunque no necesariamente suficientes, para llevar a cabo un proyecto de investigación. Con la intención de paliar este déficit, hace más de 12 años los representantes de las distintas comisiones de docencia y asesoras, ahora integradas en la Red de Comisiones de Docencia y Comisiones Asesoras de la Comunidad Autónoma de Galicia (REDEGA), de común acuerdo con el SERGAS creyeron conveniente implementar un programa formativo, denominado programa de formación común complementario, para potenciar y financiar la formación en áreas de conocimiento escasamente contempladas o desarrolladas en la carrera universitaria. Entre ellas, con un papel prominente, la metodología de la investigación en clínica y epidemiología, con una duración recomendada de 40 horas. Esta actividad formativa se considera de carácter obligatorio y, por lo tanto, debe ser exigible por cada residente en formación y exigida como requisito curricular imprescindible por los comités de evaluación antes de finalizar la residencia. El momento en que el residente debe llevar a cabo esta actividad lo fija cada comisión de docencia. No obstante, dado que los dos o tres primeros años de la residencia están condicionados por el aprendizaje de habilidades y competencias clínico-asistenciales y la participación en trabajos o proyectos de investigación es anecdótica, lo aconsejable sería realizar esta actividad en el tercer año o posteriores, puesto que la implicación y motivación son mayores y la aplicación práctica es inmediata, especialmente si hay un proyecto de tesis doctoral en marcha. En cualquier caso, y de forma análoga a la asistencia, a investigar se aprende investigando.

El segundo punto es la importancia o tema de investigación, y siguiendo una de las máximas del taoísmo, el más largo camino comienza siempre por el primer paso. Empezar con la descripción de casos clínicos relevantes por su peculiaridad, enseñanza o ejemplificación, seguir con la comunicación de pequeñas series y acabar publicando originales es una senda que han recorrido la mayor parte de los que hoy consideramos investigadores líderes en nuestro país. Investigar en clínica es difícil, porque se tiene la falsa sensación de que todo está dicho o hecho y hay poco que aportar. La investigación básica requiere además de fuentes de financiación. Sin embargo, lo realmente difícil es tener ideas y estas suelen tener que ver con el estudio, la observación y con cuestionarse continuamente lo que se realiza de forma cotidiana. Sirvan como ejemplos los profundos cambios acontecidos en los últimos años en el manejo de la enfermedad tromboembólica y de la endocarditis infecciosa, debido a que alguien se preguntó por qué estos pacientes tenían que estar ingresados y durante cuánto tiempo. Pero ser pionero sólo está al alcance de pocos y eso no quiere decir que no se pueda o deba investigar. $Y$ es que cuando se tiene una experiencia clínica amplia sobre un tema es una obligación ética comunicarla y difundirla para poder contrastar o dar réplica a lo comunicado por otros autores. En este sentido, en el ámbito de la gestión clínica en nuestra comunidad tenemos hospitales pioneros en la creación de unidades (corta estancia, hospitalización a domicilio, insuficien- 
cia cardiaca y otras) que han supuesto un cambio, incluso en algunos casos me atrevería a decir una revolución, respecto al manejo tradicional de ciertas patologías y que, en cierto modo, tienen contraída la obligación de comunicar al resto de la comunidad científica y al organismo gestor su experiencia, máxime en tiempos como los actuales caracterizados por la búsqueda de la máxima eficiencia en la gestión de recursos. Por lo tanto, la dificultad sobre qué investigar o por qué investigar debe ser entendida más como una excusa que como un argumento convincente. Sin en el servicio docente no hay líneas de investigación, en el entorno inmediato existen grupos consolidados, integrados en redes de colaboración, de fácil acceso, dotadas de plataformas tecnológicas, y que además están interesados en la colaboración con otros grupos.

Otra de las barreras esgrimidas para no investigar es la presión o sobrecarga asistencial. Evidentemente, cuando la actividad asistencial es muy alta y consume muchas energías, la motivación para investigar disminuye de una forma inversamente proporcional. En este sentido, la actividad asistencial de un facultativo gallego de medicina interna dista mucho de ser la ideal pero, como veremos en un próximo número de Galicia Clínica, es muy similar entre hospitales de las mismas características. Sin embargo, la producción científica puede diferir mucho entre ellos, como se pudo apreciar en alguna de las ofertas públicas de empleo, hecho que suele estar relacionado con la cultura de trabajo o nivel de autoexigencia de los diferentes servicios puesto que, a diferencia de otros países, nadie o nada obliga a investigar.

Investigar en grupos con la suficiente masa crítica y con líneas de trabajo bien definidas es, innegablemente, más fácil. Pero esto sólo se consigue publicando y acreditando una buena trayectoria investigadora. Cuando los méritos son suficientes es más fácil concurrir y conseguir financiación pública (becas FISS, XUGA,..) o privada (ensayos clínicos o registros) y, subsecuentemente, poder beneficiarse de reducción de la actividad asistencial mediante los denominados programas de intensificación investigadora o contratar personal investigador. En la situación actual, con las dificultades presupuestarias existentes y la previsiblemente escasa contratación, esta última podría ser una salida razonable y aconsejable para los residentes que terminan la especialidad.
Por último, en nuestra modesta opinión, el principal problema es el escaso reconocimiento curricular que tiene la investigación en nuestra profesión. Si realmente se quiere potenciar la investigación hay que poner el valor en alza. Esto conlleva un cambio de mentalidad muy marcado que debe empezar ya durante la residencia, modificando el concepto expresado en la orden ministerial arriba citada "debe saber" por el de "tiene que hacer" un proyecto de investigación. Esta exigencia para obtener el título de especialista es una forma de acreditar competencia en esta área, de manera análoga a lo que ocurre en otras profesiones como arquitectura, y debería ser sometida a un proceso de auditoría de forma regular. Asimismo, y aceptando la baja popularidad que puede provocar la idea, creo que se debería reevaluar el peso curricular de la actividad investigadora, tanto en las oposiciones como en la carrera profesional, frente a conceptos más ligados o provenientes del ámbito sindical como la antigüedad y la asistencia a cursos $u$ otras actividades formativas: sólo quien publica o realiza una tesis doctoral conoce el esfuerzo y tiempo consumidos en el empeño. El cumplimiento de este requisito tendría como consecuencias el interés de los servicios docentes por crear líneas de investigación, participar en proyectos multidisciplinares o multicéntricos promovidos por los grupos de trabajo de la sociedad o la industria farmacéutica, desarrollando una cultura de colaboración y asociación, una mayor producción científica con la subsecuente visibilidad en los diversos foros de la sociedad y, en último término, una mejora de la calidad asistencial, desterrando la falsa y poco fundamentada idea del antagonismo entre asistencia e investigación. En palabras del prestigioso doctor Joan Rodés: "Sin investigación de calidad no hay asistencia de calidad y viceversa".

\section{Bibliografía}

1. ORDEN SCO/227/2007, de 24 de enero, por la que se aprueba y publica el programa formativo de la especialidad de Medicina Interna. Boletín Oficial del Estado núm. 033, 7 de Febrero de 2007. Disponible en: http://www.boe.es/boe/ dias/2007/02/07/pdfs/A05755-05759.pdf

2. Iñiguez I, Rubal D, Matesanz M, Ventura P, Rabuñal R, Monte R. Actividad investigadora de los residentes de medicina interna de Galicia. Galicia Clin 2012; 73 (1): 7-10

3. Rabuñal R, Monte R, Paz F, Bal M, López S, Arias C et al. Análisis de la actividad investigadora de los internistas gallegos a partir de los resultados de la oferta pública de empleo. An Med Interna (Madrid) 2007; 24: 365-8.

4. Gimenez-Gómez N, Pedrazas D, Medina E, Dalmau D et al. Formación en investigación: autopercepción de los profesionales sobre sus necesidades. Med Clin (Bar) 2009; 132(3):112-117.

5. Meijide H, Rabuñal R, De la Iglesia F. La formación de residentes de medicina interna en Galicia: encuesta de opinión. Galicia Clin 2010; 71: 153-9. 